\title{
Legendre transform in the thermodynamics of flowing polymer solutions
}

\author{
J. Casas-Vázquez, ${ }^{1}$ L. F. del Castillo, ${ }^{2}$ D. Jou, ${ }^{1,3}$ and M. Criado-Sancho ${ }^{4}$ \\ ${ }^{1}$ Departament de Física, Universitat Autònoma de Barcelona, 08193 Bellaterra, Catalonia, Spain \\ ${ }^{2}$ Instituto de Investigaciones en Materiales, UNAM, Apartado Postal 70-360, México DF 04510, Mexico \\ ${ }^{3}$ Institut d'Estudis Catalans, Carme 47, 08001 Barcelona, Catalonia, Spain \\ ${ }^{4}$ Departamento de Ciencias y Técnicas Físicoquímicas, UNED, Senda del Rey s/n, 28040 Madrid, Spain
}

(Received 19 July 2000; revised manuscript received 18 December 2000; published 11 April 2001)

\begin{abstract}
We propose a Legendre transform linking two different choices of nonequilibrium variables (viscous pressure tensor and configuration tensor) in the thermodynamics of flowing polymer solutions. This may avoid some current confusions in the analysis of thermodynamic effects in polymer solutions under flow.
\end{abstract}

In this Brief Report, we propose a Legendre transform linking two different formulations of nonequilibrium thermodynamics of flowing polymer solutions, one of them depending on the viscous pressure tensor and the other one on the configuration tensor. The lack of a detailed comparison between these different possibilities has hindered the use of the nonequilibrium chemical potential, because the theoretical expressions obtained in these different choices appear to be mutually incompatible, and has caused some confusion in the analysis of thermodynamic effects in polymer solutions under flow, such as shear-induced diffusion or shear-induced shift in the critical point $[1-8]$. Here, we clarify the relation between the chemical potentials dependent on these two choices of nonequilibrium variables, and show how to use this valuable tool of equilibrium thermodynamics in the study of flowing polymer solutions, where it has still not been used because approaches beyond local equilibrium are required, where concepts of equilibrium thermodynamics must be generalized in a nontrivial way.

The chemical potential plays a central role in the analysis of polymeric solutions under flow, not only from a thermodynamic perspective $[3,5,6]$, but also from a dynamical one $[2,9]$. Indeed, to mention only one well known example, the dynamical equation describing the evolution of the concentration $c$ is, according to Helfand and Fredrickson [9],

$$
\frac{\partial c}{\partial t}+\mathbf{u} \cdot \boldsymbol{\nabla} c=\frac{\lambda}{\kappa_{B} T} \boldsymbol{\nabla} \cdot(c \boldsymbol{\nabla} \mu)+\frac{\lambda}{\kappa_{B} T} \boldsymbol{\nabla} \boldsymbol{\nabla}: \mathbf{P}^{\nu},
$$

where $\lambda$ is related to the diffusion coefficient $D$ by $D=\left(\lambda / k_{B} T\right) c, k_{B}$ is the Boltzmann constant, $\mu$ is the chemical potential of the polymer, and the balance equation for the linear momentum $\rho \mathbf{u}$ is taken to be

$$
\rho \frac{\partial \mathbf{u}}{\partial t}=-\rho \mathbf{u} \cdot \boldsymbol{\nabla} \mathbf{u}+\eta_{0} \nabla^{2} \mathbf{u}-\boldsymbol{\nabla} \cdot \mathbf{P}^{\nu}+(\boldsymbol{\nabla} c) \mu .
$$

Out of equilibrium, one cannot rely directly on classical thermodynamic stability requirements, and one analyzes the dynamical stability of Eqs. (1) and (2), complemented with an evolution equation for $\mathbf{P}^{\nu}$ as given, for instance, by the upper-convected Maxwell model [10]. To close this set of equations, an equation of state for the chemical potential is necessary.
Our first aim is to explore the form of the nonequilibrium free energy for a polymer solution, when one takes as a nonequilibrium variable the viscous pressure tensor $\mathbf{P}^{\nu}$ or, alternatively, the macromolecular configuration tensor $\mathbf{W}$, defined as $\mathbf{W}=\Sigma_{i} \mathbf{W}_{i}$, with

$$
\mathbf{W}_{i}=\frac{H}{k_{B} T}\left\langle\mathbf{Q}_{i} \mathbf{Q}_{i}\right\rangle-\frac{1}{3} \mathbf{U}
$$

where $\mathbf{Q}_{i}$ are the vectors related to the $i$ th normal mode of a macromolecule, $H$ the elastic constant of the springs in the bead-and-spring model of macromolecules, and $\mathbf{U}$ the unit tensor. Recall that $\mathbf{P}_{i}^{\nu}$, the contribution of the $i$ th mode of the macromolecule to the viscous pressure, and $\mathbf{W}_{i}$ are related for a dilute polymer solution through the Kramers relation [10] $\mathbf{P}_{i}^{\nu}=-\mathcal{G}_{i} \mathbf{W}_{i}=-J_{i}^{-1} \mathbf{W}_{i}, \mathcal{G}_{i}$ being the elastic modulus, which in the dilute concentration regime is $\mathcal{G}_{i}=n k_{B} T$, with $n$ the number of macromolecules per unit volume of the solution. The reciprocal of $\mathcal{G}_{i}$ is the steady-state compliance $J_{i}$, given by $J_{i}=\tau_{i} / \eta_{i}$, with $\tau_{i}$ and $\eta_{i}$ the relaxation time and the viscosity corresponding to the $i$ th normal mode of the macromolecule.

Both $\mathbf{P}^{v}$ and $\mathbf{W}$ have often been used as nonequilibrium variables: the first one in extended irreversible thermodynamics (EIT) [4,11-13], and the second one in theories with internal variables $[14,15]$. Each choice has its own motivations and advantages, and therefore, it is logical to try to relate them. In particular, we want to clarify the form of the free energy and of the chemical potential.

In equilibrium thermodynamics, several choices of variables may contain the whole information on the system, provided that one uses the suitable thermodynamic potential [16]: the internal energy $U(S, V, N)$, when $S$ (entropy), $V$ (volume), and $N$ (number of molecules) are taken as variables; the Helmholtz free energy $F(T, V, N)$ when $T$ (absolute temperature) is used as variable instead of $S$; or the Gibbs free energy $G(T, p, N)$, when $S$ and $V$ are replaced by $T$ and $p$ (pressure). These thermodynamic potentials are connected by Legendre transforms, which allow one to pass from one choice of variables to another without losing information. However, information is lost if thermodynamic functions are not expressed in terms of their natural variables, as for instance $S(T, p, N)$, or $F(T, p, N)$. 
Here, we examine a Legendre transform connecting a nonequilibrium free energy $F_{1}\left(T, V, N, V \mathbf{P}^{\nu}\right)$ depending on the viscous pressure tensor $\mathbf{P}^{\nu}$, and a nonequilibrium free energy $F_{2}(T, V, N, \mathbf{W})$ depending on the macromolecular configuration tensor $\mathbf{W}$. The first variable is more macroscopic than the second one, and is especially suited for the description of nonequilibrium steady states, whereas the second one is more useful for a microscopic understanding of the problem. To simplify the analysis, we consider only one normal mode of the macromolecule and a dilute polymer solution. According to EIT, the Gibbs equation in nonequilibrium has the form [11]

$$
d U=T d S-p d V+\mu d N-\frac{\mathbf{W}}{2}: d\left(V \mathbf{P}^{\nu}\right),
$$

where we have used the relation $\mathbf{P}^{\nu}=-J^{-1} \mathbf{W}$ to write explicitly the conjugate of $V \mathbf{P}^{\nu}$. We can thus write for the free energy $F_{1}$ the expression

$$
\begin{aligned}
F_{1}\left(T, V, N, V \mathbf{P}^{\nu}\right) \equiv & U-\frac{\partial U}{\partial S} S=U\left(T, V, N, V \mathbf{P}^{\nu}\right) \\
& -T S\left(T, V, N, V \mathbf{P}^{\nu}\right),
\end{aligned}
$$

in which $S$ has been replaced by $T$ as independent variable. If, instead of $V \mathbf{P}^{\nu}, \mathbf{W}$ is preferred as the independent variable, the corresponding free energy $F_{2}$ would be

$$
\begin{aligned}
F_{2}(T, V, N, \mathbf{W}) & \equiv U-\frac{\partial U}{\partial S} S-\frac{\partial U}{\partial\left(V \mathbf{P}^{\nu}\right)}: V \mathbf{P}^{\nu} \\
& =F_{1}(T, V, N, \mathbf{W})+\frac{1}{2} \mathbf{W}: V \mathbf{P}^{\nu} .
\end{aligned}
$$

The use of the adequate expression for the free energy is essential to obtain correct results for the chemical potential. Indeed, in equilibrium thermodynamics it is well known that [16] $\mu=(\partial F / \partial N)_{T, V}=(\partial G / \partial N)_{T, p}$, but, in contrast, $\mu$ $\neq(\partial F / \partial N)_{T, p}$. Similarly, in the presence of a viscous flow the chemical potential would be given by

$$
\mu=\left(\frac{\partial F_{1}\left(T, V, N, V \mathbf{P}^{\nu}\right)}{\partial N}\right)_{T, V, V \mathbf{P}^{\nu}}=\left(\frac{\partial F_{2}(T, V, N, \mathbf{W})}{\partial N}\right)_{T, V, \mathbf{W}},
$$

but

$$
\mu \neq\left(\frac{\partial F_{1}(T, V, N, \mathbf{W})}{\partial N}\right)_{T, V, \mathbf{W}}
$$

It follows that both $V \mathbf{P}^{\nu}$ and $\mathbf{W}$ can play the role of independent variables in the definition of the chemical potential, provided one uses a correct expression for the free energy. Unfortunately, misunderstandings about the definition of $\mu$ in nonequilibrium situations $[5,17]$ have been very influential in the literature and constitute a serious difficulty to assess the validity of thermodynamics in nonequilibrium steady states.

To explicitly illustrate this discussion, consider, in accordance with the integrated form of Eqs. (4) and (5),

$$
F_{1}\left(T, V, N, V \mathbf{P}^{\nu}\right)=F_{\text {eq }}(T, V, N)+\frac{1}{4} J V \mathbf{P}^{\nu}: \mathbf{P}^{\nu},
$$

where $F_{\text {eq }}(T, V, N)$ stands for the local-equilibrium free energy. Also, taking into account Eq. (6), the corresponding expression for the free energy $F_{2}$ is

$$
\begin{aligned}
F_{2}(T, V, N, \mathbf{W}) & =F_{1}(T, V, N, \mathbf{W})-\frac{\partial U}{\partial\left(V \mathbf{P}^{\nu}\right)}: V \mathbf{P}^{\nu} \\
& =F_{1}(T, V, N, \mathbf{W})+\frac{1}{2} \mathbf{W}: V \mathbf{P}^{\nu}
\end{aligned}
$$

or, using Eq. (9),

$$
F_{2}(T, V, N, \mathbf{W})=F_{\text {eq }}(T, V, N)-\frac{1}{4} J^{-1} \mathbf{W}: V \mathbf{W} .
$$

In contrast, if one writes directly $F_{1}$ in terms of $\mathbf{W}$, i.e., if one simply expresses $V \mathbf{P}^{\nu}$ in terms of $\mathbf{W}$ in Eq. (9), one gets

$$
F_{1}(T, V, N, \mathbf{W})=F_{\text {eq }}(T, V, N)+\frac{1}{4} J^{-1} V \mathbf{W}: \mathbf{W} .
$$

Note the different sign in the nonequilibrium term in Eqs. (11) and (12), which yield therefore opposite predictions for the nonequilibrium contributions.

The corresponding expressions for the chemical potential will be

$$
\mu=\left(\frac{\partial F_{1}}{\partial N}\right)_{T, V, V \mathbf{P}^{\nu}}=\mu_{\mathrm{eq}}+\frac{1}{4}\left(\frac{\partial J}{\partial N}\right)_{T, V} V \mathbf{P}^{\nu}: \mathbf{P}^{\nu}
$$

and

$$
\mu=\left(\frac{\partial F_{2}}{\partial N}\right)_{T, V, \mathbf{W}}=\mu_{\mathrm{eq}}-\frac{1}{4}\left(\frac{\partial J^{-1}}{\partial N}\right)_{T, V} V \mathbf{W}: \mathbf{W} .
$$

It is easy to see that Eqs. (13) and (14) coincide. In contrast, these results are different from those obtained by direct differentiation of Eq. (12), namely

$$
\left(\frac{\partial F_{1}(T, V, N, \mathbf{W})}{\partial N}\right)_{T, V, \mathbf{W}}=\mu_{\mathrm{eq}}+\frac{1}{4}\left(\frac{\partial J^{-1}}{\partial N}\right) V \mathbf{W}: \mathbf{W} .
$$

The chemical potential (15) is incorrect and the qualitative trends predicted on the shear-induced shift of the critical temperature are opposite to experimental observations, which are well described with the correct form (13) or (14) of the chemical potential.

Here we have discussed how the two most usual choices of the nonequilibrium variables (the configuration tensor and the viscous pressure tensor) are related through a Legendre transform; although this concept is well known in equilibrium [16], to our knowledge, it is proposed here for the first time in the context of nonequilibrium variables in flowing polymer solutions. In the literature, different forms of free energy with nonequilibrium contributions are found. How- 
ever, since not enough attention has been paid to the variables used for it, these forms do not always lead to correct expressions for the chemical potential in such a way that the predictions reached with some forms of the free energy are opposite to the predictions reached with the same free energy written in terms of other variables, but without the necessary Legendre transform. The clarification of this point is essential to progress in this field.
We acknowledge the financial support of the Dirección General de Investigación Científica y Técnica of the Spanish Ministry of Science and Technology under Grant No. BFM2000-0351-C03-01, of the Direcció General de Recerca of the Generalitat of Catalonia under Contract No. 1999SGR00095, and of the Dirección General de Asuntos del Personal Académico of the UNAM (México) under Grant No. IN-119200.
[1] U. S. Agarwal, A. Dutta, and R. A. Mashelkar, Chem. Eng. Sci. 49, 1693 (1994).

[2] A. Onuki, J. Phys.: Condens. Matter 9, 6119 (1997).

[3] D. Jou, J. Casas-Vázquez, and M. Criado-Sancho, Adv. Polym. Sci. 120, 207 (1995); M. Criado-Sancho, D. Jou, and J. CasasVázquez, Polymer 36, 4107 (1995); Phys. Rev. E 56, 1887 (1997).

[4] D. Jou, J. Casas-Vázquez, and M. Criado-Sancho, Thermodynamics of Fluids under Flow (Springer, Berlin, 2000).

[5] C. Rangel-Nafaile, A. B. Metzner, and K. F. Wissbrun, Macromolecules 17, 1187 (1984).

[6] B. Wolf, Macromolecules 17, 615 (1984).

[7] N. Clarke and T. C. B. McLeish, Phys. Rev. E 57, R3731 (1998).

[8] H. C. Öttinger, Colloid Polym. Sci. 267, 1 (1989).

[9] E. Helfand and H. Fredrickson, Phys. Rev. Lett. 62, 2468 (1989).
[10] R. B. Bird, C. F. Curtiss, R. C. Armstrong, and O. Hassager, Dynamics of Polymeric Liquids (Wiley, New York, 1987), Vol. 2.

[11] D. Jou, J. Casas-Vázquez, and G. Lebon, Extended Irreversible Thermodynamics, 2nd ed. (Springer, Berlin, 1996); Rep. Prog. Phys. 51, 1105 (1988); 62, 1035 (1999).

[12] R. E. Nettleton and S. L. Sobolev, J. Non-Equilib. Thermodyn. 20, 205 (1995); 21, 297 (1996).

[13] L. S. García-Colín and F. J. Uribe, J. Non-Equilib. Thermodyn. 16, 89 (1991).

[14] G. A. Maugin and W. Muschik, J. Non-Equilib. Thermodyn. 19, 217 (1994); 19, 250 (1994).

[15] J. Verhas, Thermodynamics and Rheology (Kluwer, Dordrecht, 1997).

[16] H. B. Callen, Thermodynamics (Wiley, New York, 1960).

[17] A. Onuki, Phys. Rev. Lett. 62, 2472 (1989); J. Phys. Soc. Jpn. 59, 3423 (1990); 59, 3427 (1990). 